\title{
Adult gonococcal keratoconjunctivitis: early detection is key
}

\author{
Daniel Lai ${ }^{1}$, Keith Ong ${ }^{1,2,3,4}$ \\ 'Department of Ophthalmology, Northern Clinical School, University of Sydney, \\ Sydney, Australia; ${ }^{2}$ Department of Ophthalmology, Royal North Shore Hospital, \\ Sydney, Australia; ${ }^{3}$ Department of Ophthalmology, Sydney Adventist Hospital, \\ Sydney, Australia; ${ }^{4}$ Department of Ophthalmology, Chatswood Private Hospital, \\ Sydney Australia
}

\begin{abstract}
We describe a case of a 52-year-old male presenting with severe mucopurulent conjunctivitis of the right eye. Corneal ulceration and associated anterior chamber activity was noted later in the course of the disease. Neisseria gonorrhoeae was positive on polymerase chain reaction (PCR) testing earlier than traditional microscopy and culture. He was successfully treated with ceftriaxone $500 \mathrm{mg}$ intravenously and azithromycin $1 \mathrm{~g}$ orally as single doses in addition to ofloxacin ophthalmic solution $0.3 \%$ hourly to the right eye. This case highlights the need to consider the possibility of gonococcus in cases of suspected bacterial conjunctivitis, careful monitoring for corneal involvement and the importance of early detection with PCR.
\end{abstract}

Keywords: corneal perforation, gonococcal conjunctivitis, Neisseria gonorrhoeae

\section{Introduction}

Gonococcal conjunctivitis (GC) typically presents as a severe mucopurulent conjunctivitis with associated lid oedema, tenderness and often preauricular lymphadenopathy. ${ }^{1,2}$ GC may be complicated by uveitis and severe keratitis. Variable corneal findings have been described, including subepithelial and/or stromal infiltrates, marginal corneal melt and diffuse oedema. ${ }^{1,3}$ This is due to the fact that Neisseria gonorrhoeae is able to penetrate an intact cornea, causing aggressive invasion and poses a high risk of corneal perforation, reportedly within 24 hours of infection. ${ }^{3,4}$

As gonococcal infection in the eye is relatively rare, diagnosis can be delayed..$^{5}$ However, clinical outcomes are known to be related to severity of disease at the commencement of adequate therapy, thereby highlighting the importance of prompt diagnosis. ${ }^{1}$

We describe a case of adult GC with corneal involvement.

Correspondence: Daniel Lai, 38 Meredith St, Strathfield, NSW 2135, Australia.

E-mail: daniel.lai01@hotmail.com 


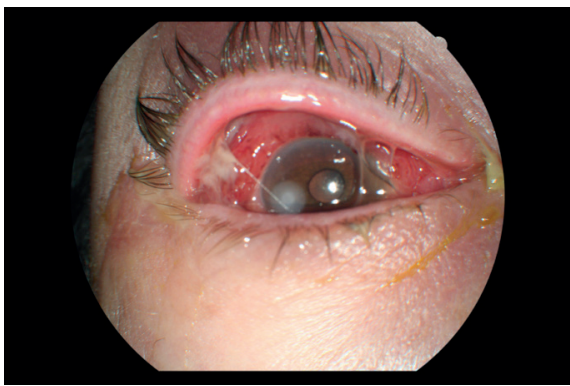

Fig. 1. Anterior segment photograph of the patient's right eye on presentation with intense conjunctival hyperaemia, chemosis and mucopurulent discharge apparent (day 1).

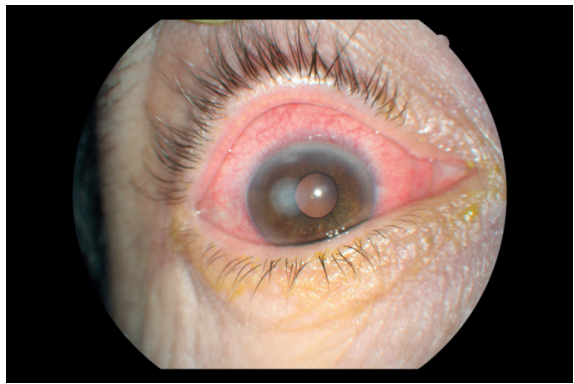

Fig. 3. Conjunctival inflammation and cornea infiltrates improving (day 5).

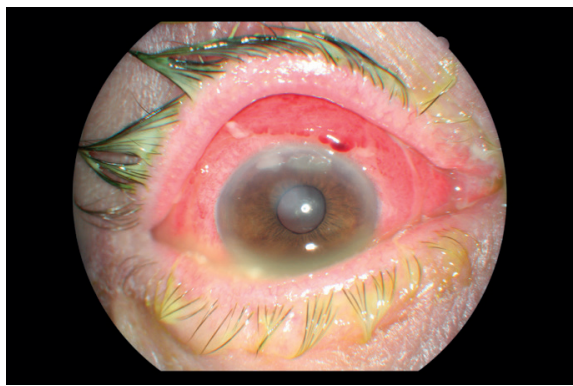

Fig. 2. Two infiltrates with overlying ulceration developing in the superior peripheral cornea evident two days later (day 3 ).

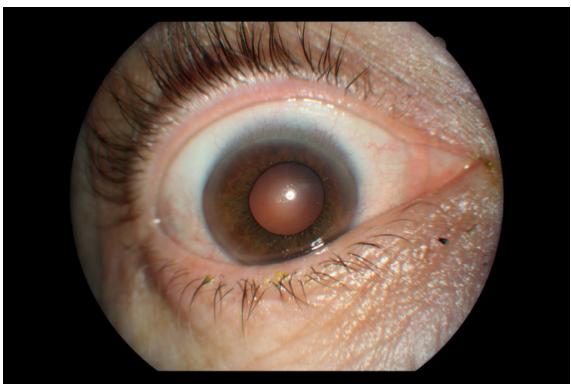

Fig. 4. Complete resolution (4 months).

\section{Case report}

The of face a 52-year-old male mechanic came into contact with debris while repairing a garbage truck. Two days later, he developed a painful eye associated with discharge and was put on chloramphenicol eye drops. His symptoms worsened and was referred to an ophthalmologist three days after the onset of his symptoms. Visual acuity was $6 / 60$ in the right eye and $6 / 9$ in the left eye; poor visual acuity was due to mucopurulent discharge and he could hardly open his right eye. On examination, there was significant inflammation of his right conjunctiva and upper eyelid with sloughing of the conjunctival epithelium epithelium (Figs. 1 and 2). The cornea was otherwise clear and anterior chamber was quiet. Conjunctival swabs were taken for microscopy, culture and sensitivity (MCS) and polymerase chain reaction (PCR) tests. A possible allergy to topical chloramphenicol was considered and his therapy was changed to oral doxycycline 100 mg daily. 
When reviewed two days later, his conjunctiva and eyelid were less oedematous. However, two small areas of corneal infiltrates with overlying ulceration had developed in his superior peripheral cornea and inflammatory cells were noted in his anterior chamber (Fig. 3). He was subsequently placed on hourly ofloxacin $0.3 \%$ ophthalmic solution (Ocuflox, Allergan).

Microscopy revealed 2+ Gram-positive cocci and 3+ leucocytes. Neisseria gonorrhoeae was positive on PCR and there was light growth on culture. Sensitivities were not provided by the laboratory. He was given azithromycin $1 \mathrm{~g}$ orally as single dose on day 4. Due to difficulties in acquiring the medication, ceftriaxone $500 \mathrm{mg}$ intravenously was administered the following day at a local hospital. On review, the corneal infiltrates and conjunctival inflammation showed significant improvement. The patient had no urethral symptoms and his partner underwent subsequent screening for sexually transmitted infections, which turned to be negative.

By day 24 , the corneal infiltrates and ulcer had resolved, with only a faint residual haze in the superior cornea and his visual acuity improved to 6/9. Complete resolution was seen four months after initial presentation (Fig. 4).

\section{Discussion}

GC can be divided into two subsets: of those affecting the adults and of those affecting the neonates, with infection traditionally considered rare in adults. ${ }^{5}$ In recent years, the incidence of gonococcal infection in Australia has increased by $118 \%$, from 35.8 per 100,000 in 2005 to 78 per 100,000 in 2015.,7 This reflects trends in the developed countries, including England and Ireland, where GC is presenting more commonly in young adults with male predominance. ${ }^{8}$

The source of the infection in this case is unclear. While most infections occur as a result of sexual contact, GC is a highly contagious condition that has several other modes of transmission. Small numbers of sporadic cases in children or adults occur every year as a result of auto-inoculation in a person with genital gonorrhoea. ${ }^{8,9} \mathrm{GC}$ can also be transmitted by direct, non-intimate interpersonal contact or contact with infected fomites (e.g. clothes, towels). ${ }^{9}$ The Australian bushfly (Musca vetustissima Walker) has also been implicated as a vector of infection. ${ }^{10}$ More rarely, epidemics can occur within a remote community or over several communities and may involve dozens or even hundreds of cases. This has occurred in central Australia four times in the past 25 years. ${ }^{11}$ Any new diagnosis raises the possibility of an epidemic occurring. Urgent notification is required, and investigation of the circumstances with a public health response is essential. Sporadic cases in children raise the possibility of sexual abuse and should be considered in patients aged less than 16 years.

Despite the well-described clinical features, incidence of gonococcal eye disease is still relatively low, leading to misdiagnosis. In one case series, all the cases of 
corneal perforation secondary to gonococcal infection were initially attributed to epidemic keratoconjunctivitis. ${ }^{12}$ Certainly in this example, a severe acute bacterial conjunctivitis, chloramphenicol allergy or chemical burn was initially considered with GC not routinely suspected. Therefore, conjunctival swabs were taken for routine MCS screening. Neisseria gonorrhoeae can be demonstrated by the presence of Gram-negative diplococci intracellularly. ${ }^{13}$ However, given that the majority of bacterial conjunctivitis are self-limiting, it may not be feasible to obtain conjunctival swabs on all cases of conjunctivitis. ${ }^{14}$ Indeed, guidelines dictate that conjunctival cultures should be reserved for suspicious cases. ${ }^{15}$ Interestingly, microscopy revealed Gram-positive cocci in this case, highlighting the significance of confirmatory tests.

Nucleic acid amplification tests (NAATs) such as PCR amplify and detect nucleic acid sequences that are specific for a particular organism. ${ }^{16}$ In theory, NAATs can produce a positive signal from as little as a single copy of the target deoxyribonucleic acid or ribonucleic acid, which makes NAATs extremely sensitive tests that decreases the time required to identify a pathogen. As such, PCR is now considered the gold standard for diagnosing GC. ${ }^{17}$ Microbiology laboratories are often inundated with specimens, and when considering that remuneration is largely government funded in Australia, cost-effectiveness is of high consideration. One such measure that would allow definitive detection could be to have a system incorporating routine PCR alongside MCS.

Current Center for Disease Control and Prevention (CDC) recommendations for uncomplicated urogenital, anorectal and pharyngeal gonorrhoea propose combination antibiotic therapy with a single dose of ceftriaxone $1 \mathrm{~g}$ intramuscularly in addition to a single dose of azithromycin $1 \mathrm{~g}$ orally or doxycycline $100 \mathrm{mg}$ twice daily orally for 7 days as presumptive treatment for concurrent Chlamydia trachomatis infection. ${ }^{18}$

Although not essential in the management of gonococcal ophthalmia, adjuvant treatment with saline lavage and topical antibiotics have been proposed. ${ }^{19}$ Topical ofloxacin $0.3 \%$ was used in this case as we strongly believe that topical therapy should be used particularly in cases of corneal involvement.

\section{References}

1. Wan WL, Farkas GC, May WN, Robin JB. The clinical characteristics and course of adult gonococcal conjunctivitis. Am J Ophthalmol. 1986;102(5):575-583.

2. Ullman S, Roussel TJ, Culbertson WW, et al. Neisseria gonorrhoeae keratoconjunctivitis. Ophthalmology. 1987;94(5):525-531.

3. Duke-Elder S. Diseases of the outer eye. In: System of Ophthalmology Vol. 8. Part 1. St. Louis: CV Mosby Co; 1965:167-174.

4. Watt PJ. Pathogenic mechanisms of organisms virulent to the eye. Trans Ophthalmol Soc UK. 1986;105:26-31. 
5. Lee JS, Choi HY, Lee JE, Lee SH, Oum BS. Gonococcal keratoconjunctivitis in adults. Eye. 2002;16(5):646-649.

6. Lahra M. Australian Gonococcal Surveillance Programme annual report, 2012. Commun Dis Intell Q Rep. 2013;37(3):E86-E87.

7. Australian Government Department of Health. National notifiable diseases surveillance system dataset. http://www9.health.gov.au/cda/source/cda-index.cfm. Accessed 2016.

8. McAnena L, Knowles SJ, Curry A, Cassidy L. Prevalence of gonococcal conjunctivitis in adults and neonates. Eye. $2015 \mathrm{Jul}$ 1;29(7):875-880.

9. Coats DK, Carothers TS, Brady-McCreery K, Paysse EA. Ocular infectious diseases. In: Feigin RD, Demmler GJ, Cherry JD, Kaplan SL, (Eds). Textbook of Pediatric Infectious Diseases. 5th ed. New York: Saunders; 2004.

10. Weinstein P. The Australian bushfly (Musca vetustissima Walker) as a vector of Neisseria gonorrhoeae conjunctivitis. Med J Aust. 1991 Nov;155(10):717.

11. Matters R, Wong I, Mak D. An outbreak of non-sexually transmitted gonococcal conjunctivitis in Central Australia and the Kimberley region. Commun Dis Intell. 1998;22(4):52-56.

12. Kawashima M, Kawakita T, Den S, Tomita M, Shimazaki J. Surgical management of corneal perforation secondary to gonococcal keratoconjunctivitis. Eye. 2009;23(2):339-344.

13. Ng LK, Martin IE. The laboratory diagnosis of Neisseria gonorrhoeae. Can J Infect Dis Med Microbiol. 2005;16(1):15-25.

14. Azari AA, Barney NP. Conjunctivitis: a systematic review of diagnosis and treatment. JAMA. 2013 Oct 23;310(16):1721-1730.

15. American Academy of Ophthalmology. Cornea/External Disease Panel. Preferred Practice Pattern Guidelines: Conjunctivitis-Limited Revision. San Francisco, CA: American Academy of Ophthalmology; 2011.

16. McElnea E, Stapleton P, Khan S, Stokes J, Higgins G. Challenges in the management of Neisseria gonorrhoeae keratitis. Int Ophthalmol. 2015 Feb 1;35(1):135-140.

17. Papp JR, Schachter J, Gaydos CA, Van Der Pol B. Recommendations for the laboratory-based detection of Chlamydia trachomatis and Neisseria gonorrhoeae-2014. MMWR Recomm Rep. 2014;63(no RR-2):1-19.

18. Centres for Disease Control and Prevention (CDC) Update to CDC's Sexually transmitted diseases treatment guidelines, 2010: oral cephalosporins no longer a recommended treatment for gonococcal infections. MMWR Morb Mortal Wkly Rep. 2012;61(31):590-594.

19. Gerstenblith AT, Rabinowitz MP. The Wills Eye Manual. 6th ed. Philadelphia: Lippincott Williams \& Wilkins; 2012. 